\title{
Adsorption geometry and interface states: Relaxed and compressed phases of NTCDA/Ag(111)
}

\author{
P. Jakob, ${ }^{1,}{ }^{*}$ N. L. Zaitsev, ${ }^{2, \dagger}$ A. Namgalies, ${ }^{1}$ R. Tonner, ${ }^{2}$ I. A. Nechaev, ${ }^{3}$ F. S. Tautz, ${ }^{4}$ U. Höfer, ${ }^{1}$ and D. Sánchez-Portal ${ }^{3}$ \\ ${ }^{1}$ Department of Physics, Philipps-Universität Marburg, 35032 Marburg, Germany \\ ${ }^{2}$ Department of Chemistry, Philipps-Universität Marburg, 35032 Marburg, Germany \\ ${ }^{3}$ Centro de Física de Materiales CFM-MPC, Centro Mixto CSIC-UPV/EHU, 20018 San Sebastián, Spain \\ ${ }^{4}$ Peter Grünberg Institut, Forschungszentrum Jülich, 52425 Jülich, Germany
}

(Received 21 July 2016; published 26 September 2016)

\begin{abstract}
The theoretical modeling of metal-organic interfaces represents a formidable challenge, especially considering the delicate balance of various interaction mechanisms and the large size of the involved molecular species. In the present study, the energies of interface states, which are known to display a high sensitivity to the adsorption geometry and electronic structure of the deposited molecular species, have been used to test the suitability and reliability of current theoretical approaches. Two well-ordered overlayer structures (relaxed and compressed monolayers) of 1,4,5,8-naphthalene-tetracarboxylic acid dianhydride (NTCDA) on $\operatorname{Ag}(111)$ have been investigated using two-photon photoemission to derive precise interface-state energies for these closely related systems. The experimental values are reproduced by our density-functional theory (DFT) calculations with two approaches to treat dispersion interactions (semi-empirical correction DFT-D3 and parametrized functional optB88) and basis set approaches (localized numerical atomic orbitals, plane waves) with remarkable accuracy. Our results underline the trustworthiness and some of the limitations of current DFT-based methods regarding the description of geometric and electronic properties of metal-organic interfaces.
\end{abstract}

DOI: 10.1103/PhysRevB.94.125436

\section{INTRODUCTION}

Electronic devices based on organic thin films contain a number of functional units which are crucial for their proper operation. The interface to the metallic leads is one of them, as it constitutes the link between two electronic subunits with very different properties; discrete molecular levels, on the one hand, meet the continuum band structure of an inorganic solid, on the other. The layer in direct contact with the substrate (contact primer layer) thereby plays a decisive role, determining the electronic structure at these interfaces, and it has been found that distinct interface states (ISs) may form as a result of the nearby molecular layer [1-5].

Based on scanning tunneling microscopy (STM) and spectroscopy (STS) data for 3,4,9,10-perylene-tetracarboxylic acid dianhydride (PTCDA) on $\operatorname{Ag}(111)$ [1], it was concluded that this electronic state, which is found to display free-electronlike dispersion, derives from an unoccupied molecular orbital (LUMO+1, where LUMO is the lowest unoccupied molecular orbital) which has broadened and shifted down in energy due to the interaction with the $\operatorname{Ag}(111)$ substrate [3]. Dispersion then arises as a consequence of mixing with delocalized metallic states (substrate-mediated intermolecular coupling). Alternatively, on the basis of the observed short lifetime of this interface state Schwalb et al. [2] argued that this state represents a modified surface-state (SS) band that has been shifted upwards by the interaction with the molecular layer. Hybridization with unoccupied molecular orbitals may also occur, but it is not a prerequisite for a delocalized interface state to emerge.

Model calculations by Dyer and Persson [3], as well as Zaitsev et al. [4], support the "upshifted surface state"

\footnotetext{
*peter.jakob@physik.uni-marburg.de

†zaitsev@chemie.uni-marburg.de
}

model by Schwalb et al. and, for the particular system PTCDA/Ag(111), confirm mixing with the LUMO+1 band of PTCDA, which has shifted down in energy upon contact with $\mathrm{Ag}(111)$. In particular, calculations in Ref. [3] reveal that outside the surface, where STM and STS are measured, the IS indeed exhibits a density distribution that clearly reflects its hybridization with the molecular orbitals. Moreover, Marks et al. [5] find that, in agreement with the original conjecture of Ref. [1], the bending of the carboxylic oxygen atoms towards the $\operatorname{Ag}(111)$ surface enhances the molecular overlap with the upshifted surface state. Note that the nature of the IS as an upshifted SS quite naturally explains its free-electronlike dispersion. More detailed calculations [6,7] have been conducted to illuminate the dependence of the IS energy $E_{I S}$ on the specific arrangement and adsorption geometry (vertical bonding distance $d_{\perp}$, bending of the molecular framework, etc.) of the adsorbed species and the surface coverage, as well as to study the expected lifetimes of the IS for various molecule-metal systems [7].

The energy position of the interface state $E_{I S}$ depends most sensitively on the vertical bonding distance $d_{\perp}$ of the molecules. For the well-studied PTCDA system $d_{\perp}$ decreases from $3.27 \AA$ for PTCDA/Au(111) [8] to $2.86 \AA$ for PTCDA/Ag(111) [9,10] and to $2.81 \AA$ for PTCDA/Ag(100) [9]. At the same time the upshift of the Shockley state $\Delta E_{I S}$ increases from $0.16 \mathrm{eV}$ [11] to $0.66 \mathrm{eV}$ [2] and to $0.95 \mathrm{eV}$ [12], respectively, resulting in an inverse scaling of $\frac{\Delta E_{I S}}{\Delta d_{\perp}}=-(1.2-1.7) \mathrm{eV} / \AA \approx-$ $1.5 \mathrm{eV} / \AA$ [13]. This high sensitivity of $E_{I S}$ on the adsorption geometry makes it an ideal model to test electronic structure calculations by comparing the results of different approaches and approximations with accurate experimental data. Despite current improvements in the description of surface and interface properties of molecular layers, the correct description of interface-state energies is by no means trivial. 
Here, in a combined experimental and theoretical approach, we investigate the well-known metal-organic model system 1,4,5,8-naphthalene-tetracarboxylic acid dianhydride (NTCDA) on $\operatorname{Ag}(111)$ to examine the dependence of interfacestate energies on the molecule-substrate binding geometry. NTCDA adsorbed on $\operatorname{Ag}(111)$ has been studied by various experimental techniques in the past [5,14-21]. Two longrange-ordered monolayer phases (relaxed and compressed monolayers, denoted r-ML and c-ML in the following) have been identified [14,15,19,21]. A moderate interaction strength of NTCDA with $\operatorname{Ag}(111)$ has been concluded based on the observed downward shift of molecular orbitals upon adsorption [18]; vibrational frequency shifts are particularly strong for the carboxyl group located at the corners of the molecule, which has been attributed to a chemical interaction with the silver substrate in addition to the ubiquitous van der Waals (vdW) interactions [19,20].

Using the normal-incidence $\mathrm{x}$-ray standing-wave technique (NIXSW), $d_{\perp}$ values have been determined for the relaxed and the compressed NTCDA monolayer phases on $\operatorname{Ag}(111)$ [16,17]. The molecular arrangements of both long-range-ordered overlayers have only very recently been resolved on the basis of low-temperature STM data [21]. For r-ML the parallel-oriented NTCDA molecules are arranged in a brick-wall-type fashion, and two inequivalent molecules within the rectangular unit cell can be distinguished. Unlike r-ML with all NTCDA aligned identically [long molecular axis oriented along $\operatorname{Ag}(111)$ atom rows], the c-ML phase comprises two different azimuthal orientations of NTCDA. More precisely, the flat-lying molecules form a herringbone structure with four inequivalent NTCDA within the unit cell.

In this context, we stress that the structural details regarding the lateral ordering are crucial to derive meaningful quantities for the vertical bonding distances, molecular deformations, adsorption energies, and electronic levels of the molecular layer from calculations and thereby allow us to establish a correlation between $E_{I S}$ and $d_{\perp}$.

The intriguing role of NTCDA/Ag(111) as a model system not only refers to the comprehensive knowledge base regarding its electronic and structural properties but additionally relies on the fact that two layers with almost identical composition (coverage, molecular orientation) exist. Specifically, the two long-range-ordered NTCDA monolayer phases differ in their coverages by only $10 \%\left(\Theta_{\mathrm{NTCDA}}=1.0\right.$ monolayer $(\mathrm{ML})$ for c-ML versus 0.9 ML for r-ML). As we will show here, these two phases exhibit interface states at well-defined but distinctly different positions, with the r-ML IS located at a higher energy. This counterintuitive result seems to stem from the balance between the effect of the molecular adsorption distance and the different coverages. Therefore, NTCDA/Ag(111) represents an ideal playground to gauge the ability of current theoretical methods based on densityfunctional theory (DFT) to predict the molecular binding geometries and the relation between those geometries and $E_{I S}$. This is particularly important since, in general, obtaining valid structural information about the molecular adsorption configuration represents an experimental challenge and the adsorption height is known from experiments only for a few selected systems (sometimes with sizable error bars). For NTCDA/Ag(111) the accumulated experimental knowledge in the literature and the data presented here for the surface electronic structure using two-photon-photoemission (2PPE) measurements pose a well-defined and stringent test to the predictive capabilities of existing DFT methods. Eventually, theory may provide trustworthy structures (via dispersion corrected DFT) and reliable $E_{I S}$ even in cases where, e.g., the experimental adsorption height is not known.

\section{EXPERIMENTAL METHODS}

The 2PPE experiments and the preparation of the sample were performed in the same ultrahigh vacuum (UHV) chamber at a base pressure below $1 \times 10^{-10}$ mbar. The $\mathrm{Ag}(111)$ crystal was cleaned by Ar-sputtering cycles $(3 \mu \mathrm{A}, 700 \mathrm{~V}, 15 \mathrm{~min}$, $373 \mathrm{~K}$ ) and subsequent annealing (773 K, $5 \mathrm{~min}$ ). The NTCDA layers were grown at a sample temperature of $90 \mathrm{~K}$ and a rate of $0.4 \mathrm{ML}$ per minute. After deposition of $\approx 1.5 \mathrm{ML}$, we prepared the c-ML and r-ML phases according to the recipe of Braatz et al. [19], i.e., by thermal annealing to either 360$370 \mathrm{~K}$ (c-ML) or $400 \mathrm{~K}$ (r-ML) and using a temperature ramp $\approx 1 \mathrm{~K} / \mathrm{s}$. The long-range order and purity of the film were checked by low-energy electron diffraction (LEED) and $\mathrm{x}$-ray photoelectron spectroscopy (XPS).

We used the laser setup described by Sachs et al. [22] (PE-I) with a pump laser energy of $\hbar \omega_{\text {pump }}=3.08 \mathrm{eV}$ (pulse width of $68 \mathrm{fs}$ ) and a probe energy of $\hbar \omega_{\text {probe }}=4.62 \mathrm{eV}$ (pulse width of $77 \mathrm{fs}$ ). The incident beams were focused onto the sample at an angle of $75^{\circ}$ with respect to the surface normal; the spectra reported here refer to a sample temperature of $90 \mathrm{~K}$ and were recorded in normal emission, i.e., at the $\Gamma$ point. Special care has been taken to avoid space-charge effects, which might adversely influence the precisely measured energetic positions. The UV beam photon energy, which was very close to the work function of the clean sample, has been reduced to a pulse energy below $0.05 \mathrm{~nJ}$ to avoid detrimental effects due to a low-energy one-photon background. The time delay has been set to zero to maximize signals.

The prominent Shockley surface state of the $\operatorname{Ag}(111)$ surface has been used to calibrate the energy scale of our experimental setup. According to Reinert et al. [23], it has a binding energy of $63 \mathrm{meV}$ at $30 \mathrm{~K}$ ( $\Gamma$ point). Paniago et al. derived a somewhat less precise value of $26 \mathrm{meV}$ at $300 \mathrm{~K}$, and more importantly, they examined the temperature dependence of this state in the temperature range 50-550 K [24]. Using the precise low- $T$ value of Reinert et al. and applying the observed $T$ dependency [Fig. 5(b) of Ref. [24]], the surface state for clean $\mathrm{Ag}(111)$ is expected at $59 \mathrm{meV}$ below the Fermi level at our sample temperature of $90 \mathrm{~K}$.

\section{CALCULATION DETAILS}

First-principles electronic structure calculations were performed in the framework of DFT using the SIESTA code [25,26], with localized numerical atomic orbitals as a basis set, and the VASP code [27], where a plane-wave expansion is used to represent the electronic wave functions. In the SIESTA code core electrons are replaced by norm-conserving Troullier-Martins-type pseudopotentials [28], while in VASP the so-called projector augmented wave (PAW) method is applied $[29,30]$. 

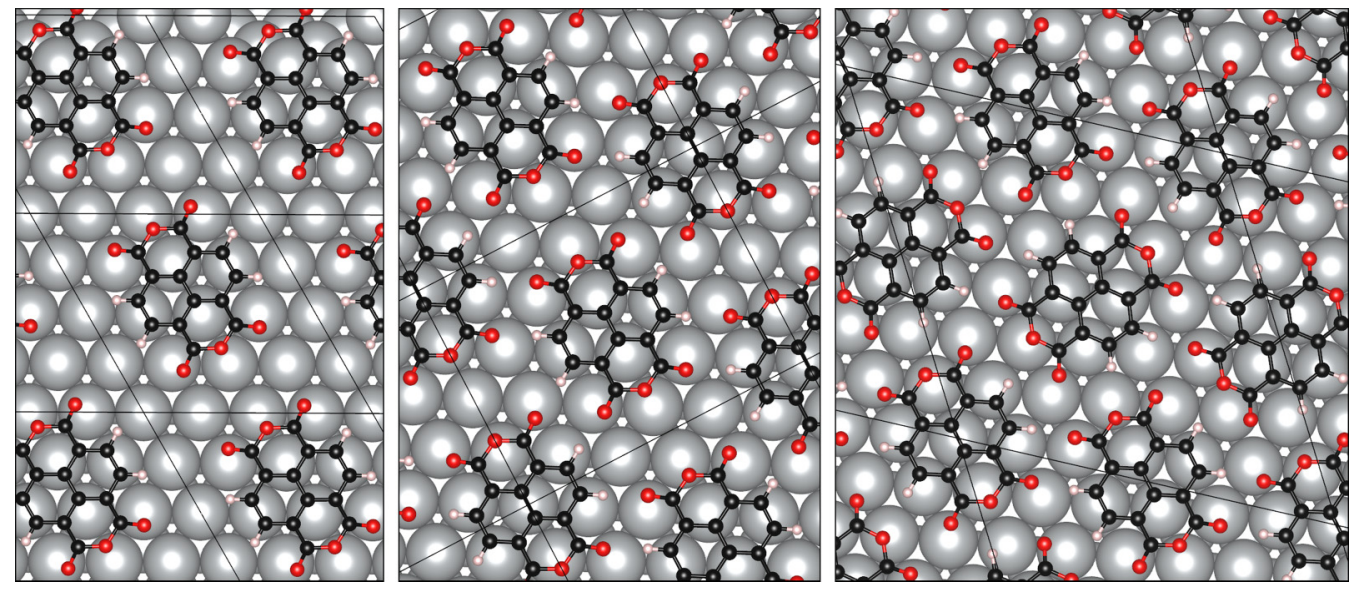

FIG. 1. The surface unit cells and initial arrangements of NTCDA molecules on Ag(111). The hypothetical $4 \times 4$ commensurate structure (left) which comprises identical adsorption geometries for all NTCDA and which is characterized by negligible intermolecular interactions has been analyzed recently by Tonner et al. [20]. The arrangement of NTCDA molecules in the experimentally observed relaxed (middle) and compressed (right) phases has been derived from low-T STM data [21].

Since local and semilocal density functionals are known to provide a poor description of the adsorption geometry of metalorganic interfaces, here we use exchange-correlation (xc) functionals that explicitly account for long-range, nonlocal dispersion interactions (vdW-DF2). In particular, we chose the optB88 functional by Klimes et al. [31,32], providing the best equilibrium lattice constant and bulk modulus for bulk silver (respectively, $4.172 \AA$ and $119 \mathrm{GPa}$ in our SIESTA calculations) among the vdW-DF2 functionals we tested. Additionally, we performed calculations within the PBE-D3 scheme [33,34], which incorporates an empirical correction to include dispersion forces on top of the generalized gradient approximation Perdew-Burke-Ernzerhof (PBE) functional [35].

A scheme of periodically repeated slabs was used to describe the $\operatorname{Ag}(111)$ surface. To avoid direct interaction between the periodic images of the system a vacuum layer of $\sim 11 \AA$ was included in the direction perpendicular to the surface ( $z$ direction). In order to get the final geometric structures, we allowed full relaxation of the NTCDA molecular monolayer and the two underlying Ag layers of the fourlayer silver slab until the maximum force became less than $0.01 \mathrm{eV} / \AA$. For the calculation of interface states we took slabs comprising 12 layers.

According to our tests, with this increased number of layers, the energy of the IS is converged within $10 \mathrm{meV}$ with respect to the slab thickness. Notice that both the SS and the IS are located within the projected band gap of $\operatorname{Ag}(111)$ but still rather close to the bulk bands and thus extend far into the substrate, requiring the use of rather thick slabs. The surface Brillouin zone was sampled using a Monkhorst-Pack scheme with eight and six $k$ points for the relaxed and compressed phase, respectively.

For SIESTA calculations, we used a double- $\zeta$ polarized (DZP) basis generated within the soft-confinement scheme. Silver orbital radii corresponded to an energy shift [26] of $180 \mathrm{meV}$, providing $3.72 \AA$ for the $5 s$ orbital. For a better description of the $\operatorname{Ag}(111)$ surface [36] we used an enlarged range of $\mathrm{Ag}$ pseudoatomic orbitals for atoms in the surface (uppermost and lowermost) layers of the slab. We explicitly checked that the position of the $\operatorname{Ag}(111)$ surface state ceases changing when the radius of the $5 s$ orbitals of $\mathrm{Ag}$ reaches a value of $5.15 \AA$, which corresponds to $10 \mathrm{meV}$ of energy shift. The same energy-shift value was used to produce the basis functions of all molecular atoms. Note that usage of extended orbitals for all the Ag atoms in the slab results in an insignificant change in the electronic properties.

A uniform mesh for numerical integration and solution of the Poisson equation was specified by an energy cutoff of $250 \mathrm{Ry}$; for structure optimization (to avoid numerical noise in the forces) this value was increased to $400 \mathrm{Ry}$. For VASP calculations, the plane-wave cutoff was fixed at $350 \mathrm{eV}$.

The unit cells and arrangements of the molecules within them are depicted in Fig. 1. The artificially diluted $(4 \times 4)$ structure contains one molecule per unit cell which is located above bridge-site positions of the silver substrate (molecule center) and with the central C-C bond aligned along $\mathrm{Ag}$ atom rows (Fig. 1, left). There are two molecules in the unit cell of r-ML. One molecule occupies a bridge site; the other one occupies the on-top position. The unit cell of c-ML contains four molecules; two of them occupy the same adsorption sites as for the relaxed monolayer phase. The remaining two molecules are rotated by about $90^{\circ}$ with respect to first ones and sit on bridge sites. The arrangements of the molecules in the r-ML and c-ML phases were deduced from high-resolution STM images [21]. The analyzed structures refer to different coverages of $0.672 \mathrm{ML}, 0.896 \mathrm{ML}$, and $1 \mathrm{ML}$ for the dilute $(4 \times 4)$, r-ML, and c-ML phases, respectively; $1 \mathrm{ML}$ is thereby defined as the maximum amount of parallel-oriented NTCDA, which can be accommodated on $\operatorname{Ag}(111)$, i.e., for the compressed monolayer.

\section{RESULTS}

\section{A. Experimental results}

Figure 2 displays 2PPE spectra of c-ML and r-ML. The NTCDA/Ag(111) sample has been prepared by NTCDA deposition at low $T \approx 80 \mathrm{~K}$ (for details, see the experimental section) and annealing to successively higher temperatures 
(a)

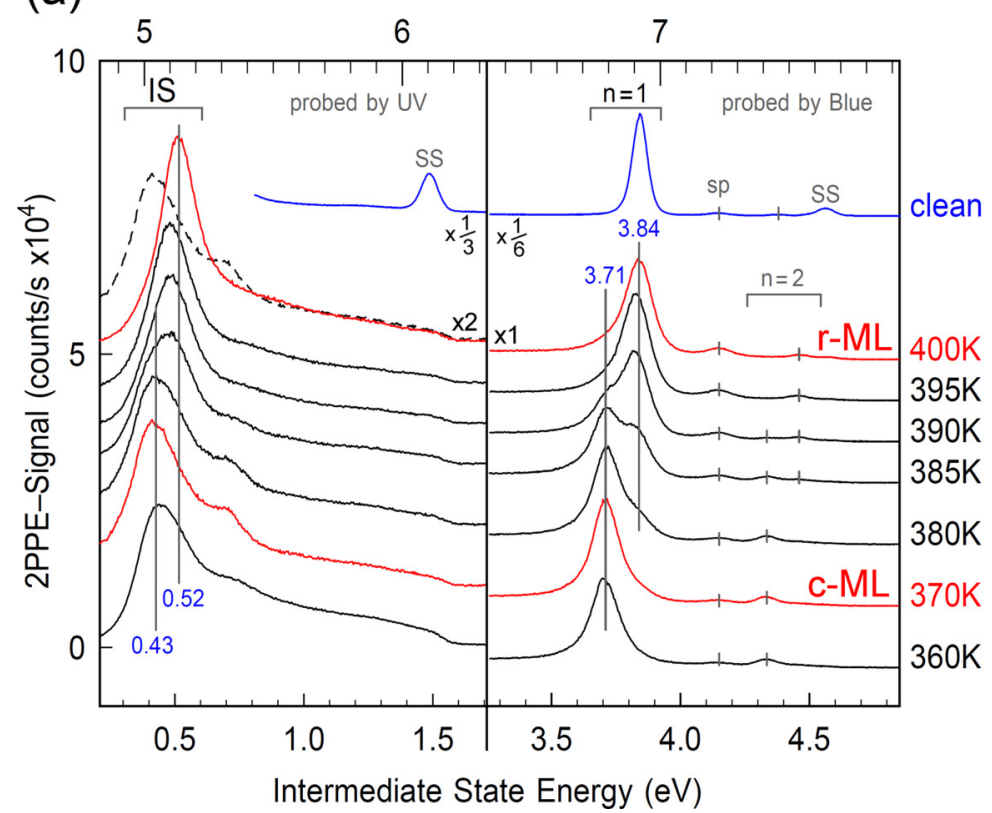

(b)

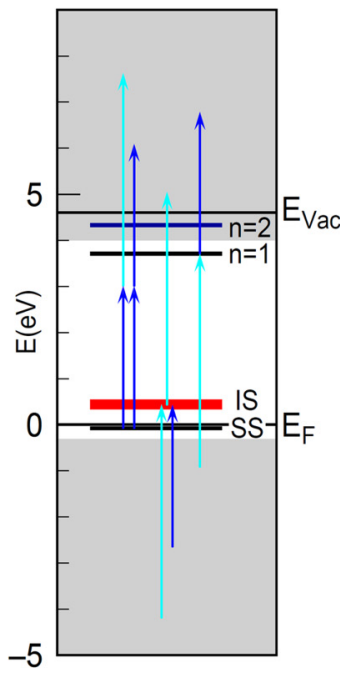

FIG. 2. (a) Series of 2PPE spectra of NTCDA on $\operatorname{Ag}(111)$ in the coverage range 0.9-1.0 ML. Starting with the compressed monolayer $\left(\Theta_{\mathrm{NTCDA}}=1.0 \mathrm{ML}\right)$, the NTCDA coverage was successively reduced by stepwise annealing until a complete relaxed monolayer $\left(\Theta_{\mathrm{NTCDA}}=0.9\right.$ ML) was obtained. The respective annealing temperatures are indicated to the right of the right panel. Note that the vertical scale displaying the NTCDA/Ag(111) interface state (left panel) has been enlarged by a factor of 2 with respect to the spectral region of image potential states (right panel). In order to aid direct comparison of the interface states for r-ML and c-ML the latter trace (dashed line) has been overlaid on the r-ML curve. The spectrum of the clean $\mathrm{Ag}(111)$ surface (topmost curve) covering the Shockley surface state (SS), the Ag bulk $s p$ band (sp), and the image-potential states $(n=1, n=2)$ has been added for comparison. Final-state energies of the emitted electrons, as well as the energies of the intermediate state (not applicable to SS, see main text), are given with respect to the Fermi energy $E_{F}$. (b) Excitation and probing scheme indicating the various spectral features of the compressed monolayer. The length of the blue and turquoise arrows is proportional to the photon energy of the blue and UV laser beams. The gray areas display the projected $\operatorname{Ag}(111)$ bulk bands at the $\Gamma$ point.

(and recooling thereafter for data collection); due to desorption of NTCDA in the temperature range $370-400 \mathrm{~K}$ the complete monolayer (c-ML phase) gradually transforms to the less dense r-ML phase. The image-potential states of the NTCDA monolayer thereby serve as an indicator for the quality and integrity of the layers. Specifically, discrete bands associated with the two phases are observed at distinctly different energies. The $n=1,2$ image-potential states of c-ML are located at 3.71 and $4.33 \mathrm{eV}$, and those of r-ML are located at 3.84 and $4.46 \mathrm{eV}$ above $E_{F}$, respectively (see Fig. 2). The spectrum of the clean silver is added on top of the figure for comparison. In contrast to the emission of the unpopulated interface and image-potential states, the populated Shockley surface state, which was used for the energetic calibration, can only be emitted by a two-photon process. It appears at the final-state energy position of 6.1 or $7.64 \mathrm{eV}$, depending on whether the emission is induced by absorption of two blue photons $\left(\hbar \omega_{\text {blue }+ \text { blue }}=6.16 \mathrm{eV}\right.$ ) or one blue and one UV photon $\left(\hbar \omega_{\text {blue }+\mathrm{UV}}=7.70 \mathrm{eV}\right)$.

The absence of any unwanted sidebands or shoulders for $n=1$ and the absence of the prominent $\operatorname{Ag}(111)$ surface state are taken as proof of the excellent quality and homogeneity of the prepared phases. Moreover, their narrow line shapes support the idea that this transition proceeds by a gradual shift of the boundary separating both phases, as has been suggested by Stahl et al. [14] based on STM data.
In the region of the interface state, i.e., $0-1 \mathrm{eV}$ above $E_{F}$, we identify distinct spectral features at $0.43 \mathrm{eV}$ (c-ML) and $0.52 \mathrm{eV}$ (r-ML). For both bands the readout proceeds exclusively via UV absorption; excitation of electrons in this unoccupied electronic level may be achieved by means of either blue or UV light.

Comparison of the IS energies for c-ML and r-ML thus reveals a notably lower value for c-ML, which may indicate a larger vertical bonding distance of NTCDA for this phase compared to r-ML. Since the NTCDA coverage is about $10 \%$ less for r-ML, an extrapolation to coverages equal to the c-ML phase would cause an extra upshift of the r-ML interface-state energy, implying an accordingly larger difference in vertical bonding distance between the c-ML and r-ML phases.

We note that for less dense layers $\left(\Theta<\Theta_{\max }\right)$ empty spaces between molecules cause leakage of the IS wave function into the vacuum, leading to a lowering of $E_{I S}$. The effect of an incomplete molecular layer has been examined theoretically for an idealized, planar layer of NTCDA on $\operatorname{Ag}(111)$ and will be discussed in more detail below. An estimate based on these findings (extrapolation to full saturation of the monolayer) would produce a $20-40 \mathrm{meV}$ upshift of the interface state, assuming a vertical bonding distance of 2.9-3.0 $\AA$. According to the projected shift of $E_{I S}$ versus vertical bonding distance of about $1.5 \mathrm{eV} / \AA$ [13], the observed $(90 \mathrm{meV})$ plus extrapolated (20-40 meV) 
TABLE I. Vertical adsorption distances [values given in $\AA$ and with respect to the extrapolated bulk positions of the Ag(111) surface atoms] for the relaxed NTCDA $/ \operatorname{Ag}(111)$ monolayer phase. The bending of the molecules is quantified by $\delta\left(C_{\text {core }}-O_{\text {carb }}\right)$. The different parts of the molecule are specified with the following notation: $C_{c}$, two central carbon atoms; $C_{\text {core }}$, four inner carbon atoms of naphthalene core; $C_{H}$, four carbon atoms connected to $\mathrm{H}$ atoms; $C_{\text {func }}$, four carbon atoms connected to carboxylic oxygens $O_{\text {carb }} ; O_{\text {anh }}$, anhydride oxygens. The computed lattice parameters $a_{A g}$ of $\mathrm{Ag}$ using each computational scheme are also indicated. The experimental value for $\mathrm{C}$ atoms corresponds to averaging over all $\mathrm{C}$ atoms for r-ML.

\begin{tabular}{|c|c|c|c|c|c|c|c|}
\hline & \multicolumn{2}{|c|}{$\begin{array}{l}\text { SIESTA: optB88 } \\
\left(a_{A g}=4.17 \AA\right)\end{array}$} & \multicolumn{2}{|c|}{$\begin{array}{c}\text { VASP: optB88 } \\
\left(a_{A g}=4.14 \AA\right)\end{array}$} & \multicolumn{2}{|c|}{$\begin{array}{l}\text { VASP: PBE-D3 } \\
\left(a_{A g}=4.07 \AA\right)\end{array}$} & \multirow{2}{*}{$\begin{array}{c}\text { Expt. }^{\mathrm{a}} \\
\left(a_{A g}=4.06 \AA^{\mathrm{b}}\right)\end{array}$} \\
\hline & Top & Bridge & Top & Bridge & Top & Bridge & \\
\hline$C_{c}$ & 3.06 & 3.09 & 3.11 & 3.12 & 3.05 & 3.10 & \\
\hline$C_{\text {core }}$ & 3.01 & 3.03 & 3.06 & 3.06 & 3.00 & 3.04 & 2.997 \\
\hline$C_{H}$ & 2.94 & 2.96 & 3.02 & 3.01 & 2.93 & 2.96 & \\
\hline$C_{\text {func }}$ & 2.86 & 2.87 & 2.92 & 2.89 & 2.88 & 2.88 & \\
\hline$O_{\text {carb }}$ & 2.69 & 2.61 & 2.73 & 2.64 & 2.69 & 2.63 & 2.747 \\
\hline$O_{\mathrm{anh}}$ & 2.85 & 2.85 & 2.92 & 2.89 & 2.91 & 2.90 & 3.004 \\
\hline $\mathrm{H}$ & 2.91 & 2.93 & 3.0 & 2.99 & 2.91 & 2.94 & \\
\hline$\delta\left(C_{\text {core }}-O_{\text {carb }}\right)$ & 0.32 & 0.42 & 0.33 & 0.42 & 0.31 & 0.41 & \\
\hline
\end{tabular}

a NIXSW [17]

${ }^{\mathrm{b}}$ Experimental value corrected for zero-point energy effects [38].

difference in interface-state energy of +(110-130) meV for $\mathrm{r}-\mathrm{ML}$ with respect to c-ML would translate into a $0.08 \pm$ $0.01 \AA$ closer average height of NTCDA above $\operatorname{Ag}(111)$ for r-ML compared to c-ML.

This value may be compared to NIXSW literature data which have been obtained for both ordered phases of NTCDA/Ag(111) [16,17]. For r-ML and c-ML, $C_{1 s}$ and $O_{1 s}$ data have been analyzed to derive adsorption heights (averaged over all C atoms) of $3.02 \pm 0.02 \AA$ for r-ML and $3.09 \pm 0.04 \AA$ for c-ML [16]. Later on, more precise values have been derived for r-ML (see Table 1) [17]. These values are notably lower than expected for pure van der Waals interacting molecular species and underline the significance of chemical interactions for NTCDA on $\operatorname{Ag}(111)$. This finding is also in accordance with a marked chemical shift in XPS binding energies [37] and the partial filling of the former LUMO of adsorbed NTCDA [18]. According to NIXSW findings for $C_{1 s}$ and $O_{1 s}$ core levels, the planar molecular structure of NTCDA becomes warped when adsorbed on $\operatorname{Ag}(111)$. This has been attributed to covalent bonding of NTCDA to substrate atoms via the four carboxyl groups, in accordance with similar findings for PTCDA on $\operatorname{Ag}(111)$ [10]. Such covalent bonding and the accompanying downward bending of carboxyl oxygens agree favorably with the pronounced redshift of respective $\mathrm{C}=\mathrm{O}$ stretching modes for NTCDA on $\mathrm{Ag}(111)$ [19-21].

In NIXSW data, as well as in our theoretical calculations, the extrapolated bulk positions of $\mathrm{Ag}(111)$ surface atoms have been used as the reference frame $\left(z_{\text {ref }}=0\right)$. True spacings between molecule and metal then additionally comprise surface relaxation effects which may add up to values of about $0.1 \AA$ and additionally depend crucially on the type of adsorbed species. For NTCDA/Ag(111) the difference between the extrapolated bulk position of $\operatorname{Ag}(111)$ and the calculated average position of Ag surface atoms amounts to $0.02 \AA$ at most for all structures considered in this study.

Analysis of adsorbed PTCDA or NTCDA on $\operatorname{Ag}(111)$ [9,20] provided clear evidence of a non-negligible relaxation of $\mathrm{Ag}$ surface atoms which not only did comprise an overall contraction of the vertical bonding distance but also introduced an extra corrugation of surface atoms underneath of PTCDA or NTCDA. As shown below, our calculations confirm that this is the case also for the NTCDA c-ML and r-ML phases discussed here.

To conclude this section, our observed IS energies for c-ML and r-ML are fully consistent with existing NIXSW data. The next step is therefore to use DFT to derive a model of both parallel-oriented NTCDA/Ag(111) phases including their detailed adsorption geometries and, moreover, to determine the corresponding molecular electronic structure and interface state energies.

\section{B. Theoretical results}

We found optimized geometries for the r-ML and c-ML phases of NTCDA on $\mathrm{Ag}(111)$ by means of VASP (plane waves) and SIESTA (numerical pseudoatomic orbitals) calculations performed with different schemes to take into account vdW interactions, the optB88 functional and the PBE functional plus D3 empirical dispersion corrections. Although these approximations provide slightly different bulk properties (e.g., the optB88 functional overestimates the equilibrium lattice constant), the final adsorption geometries are quite similar (Table I).

The calculated optimal structures of the relaxed phase are in good agreement with experimental measurements (see Table I). In accordance with NIXSW data $[16,17]$ we find that the $O_{\text {carb }}$ position is shifted down with respect to the carbon core, while $O_{\text {anh }}$ resides in the same plane as $C_{\text {func }}$. The vertical bonding distance of the $\mathrm{C}$ backbone for the bridge molecule is $0.02 \AA$ higher than the one for on-top sites. The (vertical) distortion along the long axis of NTCDA, i.e., $\delta\left(C_{\text {core }}-C_{\text {func }}\right)$, is about $0.15 \AA$, while the distortion along the short axis, i.e., $\delta\left(C_{\text {core }}-H\right)$, amounts to $0.1 \AA$ for both types of molecules. In general, the overall bending $\delta\left(C_{\text {core }}-O_{\text {carb }}\right)$ of the on-top 

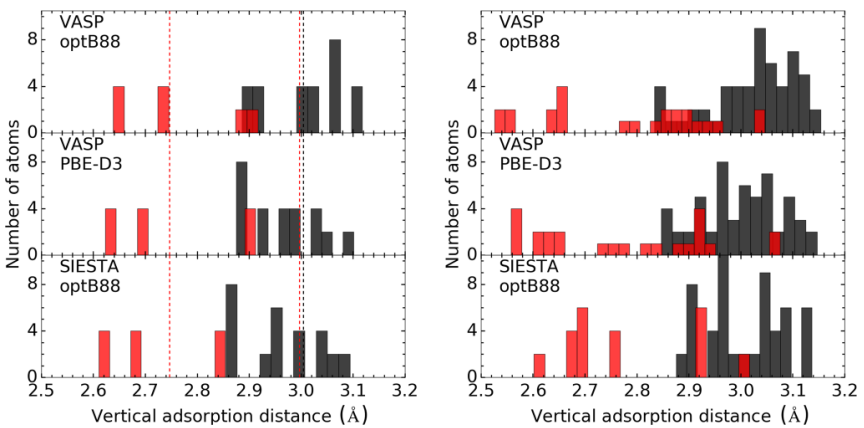

FIG. 3. The distribution of vertical adsorption distances in equilibrium geometry for the relaxed (left) and compressed (right) phases. The oxygen and carbon atoms are represented by red and black bins, respectively. According to Stanzel et al. [16], vertical heights of carbon atoms in the relaxed and the compressed phases of NTCDA on $\mathrm{Ag}(111)$ are 3.02 and 3.09 $\AA$, respectively. More recent measurements for the relaxed phase [17] are depicted as vertical dashed lines.

molecule is somewhat smaller than that of bridge-bonded NTCDA by $0.1 \AA$ (see Table I), and the respective values seem largely independent of the choice of the xc functional. As we are not sure how the averaging of individual $C_{1 s}$ signals contributes to the experimental values of carbon vertical distances $d_{\perp}$, we should not be overly concerned regarding residual differences since the overall agreement is very good.

A graphic illustration of vertical positions of the various functional groups of the NTCDA molecule within the relaxed and the compressed phases is provided in Fig. 3. Again, the three different theoretical approaches (see Table I) have been applied. In accordance with available experimental data [16], the vertical height of the carbon core is slightly larger $(\sim 0.05 \AA)$ for c-ML than for r-ML. In general, our calculations, however, display similar adsorption geometries for the two phases of NTCDA/Ag(111) (Fig. 3). In particular this applies to the aligned bridge and on-top adsorbed NTCDA molecules of c-ML, i.e., those with identical coordination with respect to the $\mathrm{Ag}(111)$ surface atoms as in the r-ML phase; interestingly, even the silver atoms underneath these NTCDA are subject to virtually the same modification (see Fig. 4). Within the c-ML the bridge and on-top molecules are slightly rotated by $2^{\circ}$ with respect to the molecules of the relaxed monolayer phase (which are strictly aligned along the $\mathrm{Ag}$ substrate atoms); in fact, our finding of a lower total energy for a slightly rotated molecular arrangement is in accordance with the local symmetry $\left(C_{2}\right)$ experienced by the individual NTCDA within the c-ML molecular layer. This is actually the reason why the bridge-bonded molecules become slightly twisted with respect to their diagonal. The remaining two inequivalent molecules within the c-ML phase, one of which lies at the center and the other of which lies at the edge of the drawn unit cell (Fig. 1), both occupy bridge sites, however, with a strong azimuthal misorientation with respect to $\mathrm{Ag}(111)$ atom rows, which leads to a twisting of their molecular planes as well.

The molecule-substrate interaction causes not only a distortion of the molecules but, additionally, a noticeable corrugation of the silver surface layer, especially for the on-top molecule. The attractive interaction of carboxylic oxygens with underneath silver atoms pulls them out from their ideal
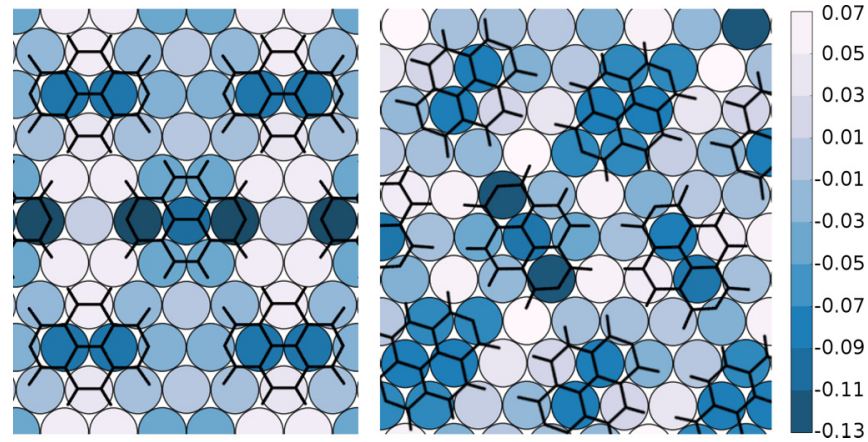

FIG. 4. The vertical deviation (in $\AA$ ) of the silver atoms in the topmost layer from their idealized positions [extrapolated bulk positions of $\operatorname{Ag}(111)$ surface atoms]. The left and right panels show the arrangement of molecules in the relaxed and the compressed NTCDA/Ag(111) monolayers, respectively. The calculation was performed using SIESTA and the optB88 functional. Positive (negative) values refer to displacements towards the vacuum (bulk).

plane. In contrast, carbon atoms on the longitudinal axis and anhydride oxygens push the underlying silver atoms downwards towards the bulk (Fig. 4), indicative of a repulsion between them. In the case of the bridge molecule we observe a similar trend but with smaller displacements. Thus, the overall interaction between the molecules and the substrate comprises two major contributions, similar to the case of PTCDA monolayers on different Ag surfaces [9]: Specifically, the derived geometry is determined by the interplay between the attraction of the functional groups with silver atoms, the repulsion of the carbon backbone from the substrate, and the ability of the molecule and the surface to accommodate such distortions.

The surface and interface state energies $\left(E_{S S}\right.$ and $\left.E_{I S}\right)$ for the r-ML and c-ML phases are presented in Table II. It is apparent that the absolute position of the interface states and the difference $\Delta E$ between the energy of SS and IS are directly dependent on the lattice parameter of the substrate. Bigger lattice constants provide higher absolute positions of SS and IS and somewhat smaller values of $\Delta E$ (Table II). Calculations performed with the same xc functional but with various choices of basis function types (and codes) give similar values for $\Delta E$. In general, it is difficult to compute the absolute positions of IS and SS accurately (slabs containing a very large number of atomic layers are required) or to determine $E_{F}$ with high precision (very dense $k$ sampling is required in combination with the large supercells used here) because very large computational resources are needed, especially in the case of plane-wave calculations.

For a meaningful description and comparison of the two NTCDA phases the applied methods of calculation should use the same parameters. Moreover, the importance of using $E_{S S}$ as a reference scale for $E_{I S}$ is apparent from Table II: Values of $E_{I S}$ are found in better agreement with the experiment in this case. The improved accuracy of thus obtained interface state energies is attributed to a compensation of (calculation) errors in deriving $E_{S S}$ and $E_{I S}$ values and the well-known energetic position (experiment) of the $\mathrm{Ag}(111)$ surface state [23,24]. 
TABLE II. The surface-state (SS) and interface-state (IS) energies for the layers displayed in Fig. 1 and calculated with different approaches. All energy values are given in $\mathrm{meV}$.

\begin{tabular}{|c|c|c|c|c|c|c|c|c|c|c|}
\hline & \multirow[b]{2}{*}{$\mathrm{xc}$} & \multicolumn{3}{|c|}{$4 \times 4$} & \multicolumn{3}{|c|}{ Relaxed ML } & \multicolumn{3}{|c|}{ Compressed ML } \\
\hline & & $E_{I S}$ & $E_{S S}$ & $\Delta E$ & $E_{I S}$ & $E_{S S}$ & $\Delta E$ & $E_{I S}$ & $E_{S S}$ & $\Delta E$ \\
\hline \multirow[t]{2}{*}{ VASP } & $\operatorname{optB} 88\left(a_{A g}=4.14 \AA\right)$ & & & & 433 & -72 & 505 & 481 & -54 & 535 \\
\hline & $\operatorname{PBE}-\mathrm{D} 3\left(a_{A g}=4.07 \AA\right)$ & & & & 424 & -164 & 588 & 450 & -157 & 607 \\
\hline \multirow[t]{2}{*}{ SIESTA } & $\operatorname{optB} 88\left(a_{A g}=4.17 \AA\right)$ & 445 & -8 & 453 & 540 & 0 & 540 & 554 & -5 & 559 \\
\hline & $\operatorname{PBE}-\mathrm{D} 3\left(a_{A g}=4.07 \AA\right)$ & & & & 468 & -137 & 605 & 480 & -153 & 633 \\
\hline
\end{tabular}

The computed values for $\Delta E$ in Table II must be compared with our experimental values (assuming $E_{S S} \simeq-59 \mathrm{meV}$ at $T=90 \mathrm{~K}$ ) of $\sim 490$ and $\sim 580 \mathrm{meV}$ for the c-ML and r-ML phases, respectively. Taking into account the difficulties in the calculations noted above, the agreement with the data in Table II can be considered quite good.

Remarkably and contrary to the experimental data, our calculations predict the IS to be somewhat higher for the compressed layer, following the usually observed trend of the IS to upshift with increasing coverage. This trend is further confirmed by the computed data presented for the more dilute hypothetical $4 \times 4$ phase. According to these data $\Delta E$ increases by $\sim 90 \mathrm{meV}$ when the coverage increases from 0.672 $\mathrm{ML}(4 \times 4)$ to $0.895 \mathrm{ML}(\mathrm{r}-\mathrm{ML})$ and by an additional $\sim 20 \mathrm{meV}$ when increasing the coverage up to $1 \mathrm{ML}$ for the c-ML phase.

Figure 5 presents the corresponding band structures for the three studied structures. We see an upshift of the IS with coverage takes place despite an enlargement of the vertical adsorption distances. Specifically, the functional group for the $4 \times 4$ phase is located distinctly closer to the substrate $\left(O_{\text {carb }}\right.$ : $2.53 \AA ; O_{\text {anh }}: 2.77 \AA$; $C_{\text {func }}: 2.8 \AA$ ) in comparison with $\mathrm{r}$ ML (see Table I). This should lead to an increase in the IS energy [6]; however, this contribution apparently is noticeably weaker compared to the effect of coverage.

The comparatively smaller increase of the IS energy when going from r-ML to c-ML is most likely due to the somewhat higher average positions of the molecules in the c-ML phase, as discussed in conjunction with Fig. 3. The larger distance to the substrate thus tends to compensate the effect of the increasing coverage. Note that the effective mass of the IS is about the same for all structures considered here $\left(\sim 0.46 m_{e}\right)$, and it is slightly bigger than the mass of the SS $\left(\sim 0.44 m_{e}\right)$.

The competition between the effect of the molecular height and that of the coverage on the IS energy is explored in more detail in Table III. Here we use a flat NTCDA monolayer, for which the molecular height over the silver surface $d_{\perp}$ can be defined unambiguously, and explore the dependence of the energy position of the IS for molecular layers arranged according to both the r-ML and c-ML phases. In this simplified model we neglect the bending of the molecules.

We note that, even for the unrelaxed layers, the optimal height of the molecules over the $\operatorname{Ag}(111)$ substrate is close to that found for the carbon molecular framework for the fully optimized structures. In general, the energy of the IS, for a given $d_{\perp}$ is always higher for the compressed ML, especially for vertical distances $d_{\perp} \leqslant 3.1 \AA$. Increasing $d_{\perp}$ to $3.2 \AA$ and beyond, on the other hand, yields only minor differences. For a fixed $d_{\perp}$ (in the range $2.9-3.3 \AA$ ), the effect of the larger coverage of c-ML produces shifts in the range from 2 to 37 meV.

Interestingly, the data in Table III indicate that, comparatively, the effect of the molecular height on the IS position is more important than that of coverage for NTCDA/Ag(111) and related systems.

Over the full range of distances explored, a change of $0.1 \AA$ produces shifts of the IS energy ranging from 47 to $67 \mathrm{meV}$ for the r-ML phase and from 49 to $85 \mathrm{meV}$ for the c-ML. Therefore, we can conclude that small height changes might easily compensate the effect of the coverage and that the effect of the molecular height is somewhat larger for the c-ML phase. Measurements $[16,17]$ indicate that the vertical adsorption distance of the carbon atoms in the relaxed monolayer phase is close to $3.0 \AA$, while it is slightly lower than $3.1 \AA$ for the compressed phase. Taking those heights and using the corresponding data in Table III (highlighted in bold), we find that this height difference is sufficient to justify a $\sim 50 \mathrm{meV}$ higher position for the IS of the r-ML phase, thus largely compensating the effect of the coverage and bringing the calculated results in qualitative agreement with the experimental observation. The failure of the DFT approaches to fully account for the experimental findings (e.g., underestimating the difference in $d_{\perp}$ for c-ML and r-ML) is probably due to the limitations of the present functionals in describing the delicate interplay between the increased intermolecular interactions in the compressed layer and the molecule-substrate interactions.

Therefore, our observation of a higher position of the IS for r-ML compared to c-ML can be interpreted as an indication

TABLE III. Interface-state energies for flat monolayers of NTCDA on an $\operatorname{Ag}(111)$ slab with 12 silver layers. The relaxed and compressed structures with different adsorption heights of the flat monolayer are presented. The vertical bonding distances $d_{\perp}$ are given relative to the position of the topmost silver layer. The interface-state energies for $d_{\perp}$ corresponding to experimental values are shown in bold.

\begin{tabular}{lcc}
\hline \hline & \multicolumn{2}{c}{$E_{I S}(\mathrm{meV})$} \\
\cline { 2 - 3 }$d_{\perp}(\AA)$ & Relaxed & Compressed \\
\hline 2.9 & 495 & 532 \\
3.0 & $\mathbf{4 2 8}$ & 447 \\
3.1 & 368 & $\mathbf{3 7 6}$ \\
3.2 & 312 & 316 \\
3.3 & 265 & 267 \\
\hline \hline
\end{tabular}



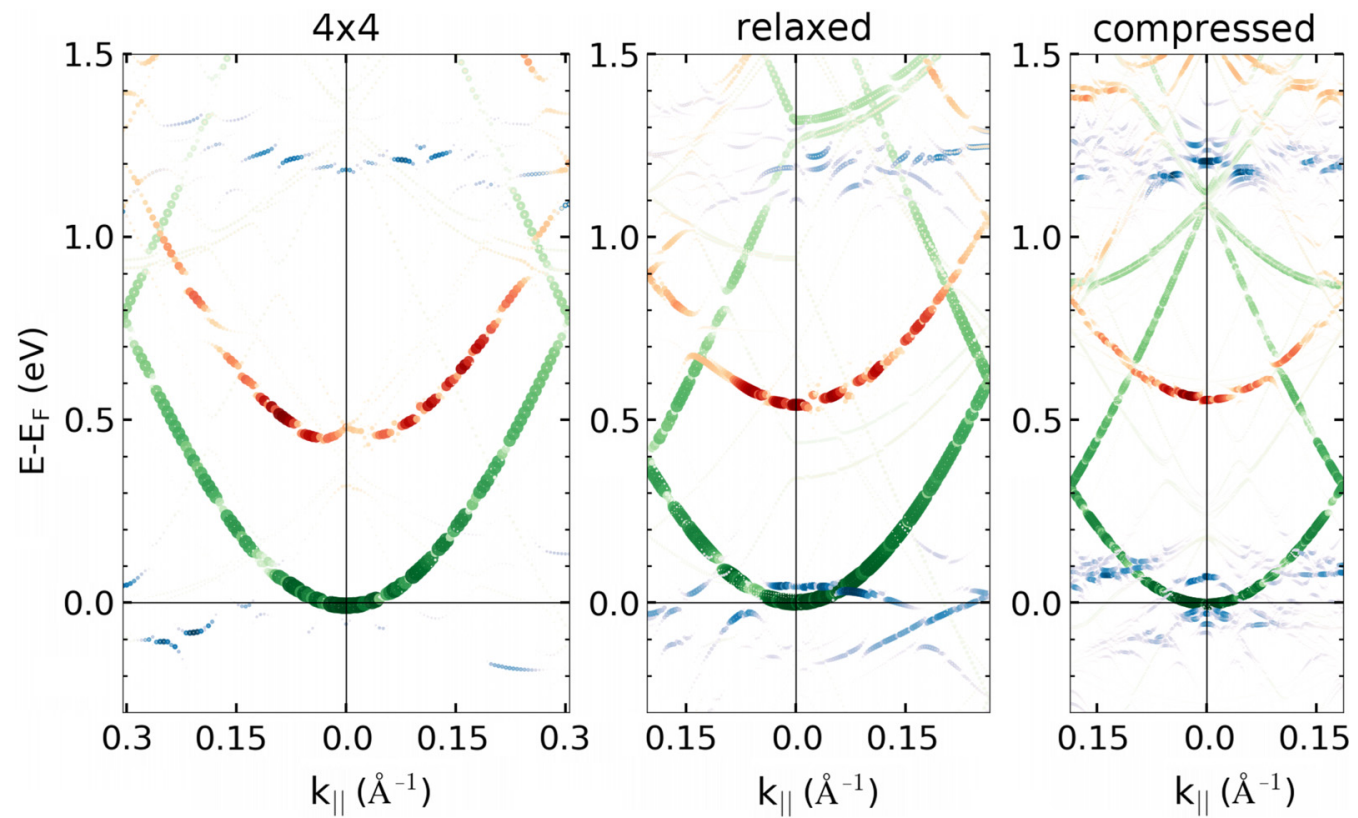

FIG. 5. Band structure of the different NTCDA/Ag(111) structures studied here using the SIESTA code, the optB88 xc functional, and a slab containing 12 layers to represent the silver substrate. The colors and intensity of the symbols indicate the weight on selected atomic orbitals. Red symbols indicate the contribution from $p_{z}$ orbitals of the topmost silver layer and reveal the interface state. Green symbols highlight the $p_{z}$ character on the silver layer from the clean side of the slab and reveal the surface state. Carbon and oxygen $p_{z}$ character are shown in blue.

that the vertical separation for c-ML is notably larger than for r-ML. This causes a compensation of the upshift of $E_{I S}$ due to coverage. According to the experimental findings, which yield a value of $E_{I S}$ which is about $90 \mathrm{meV}$ higher for r-ML than for c-ML, this compensation effect might be even larger than the estimates in the preceding paragraph. A much closer molecule-metal separation for r-ML is also consistent with the higher adsorption energy for this phase, as deduced from thermal desorption data [19].

\section{SUMMARY}

For the model system NTCDA/Ag(111), the properties of the interface state of the relaxed and compressed monolayer phases have been investigated using density-functional theory and two-photon photoemission.

The dependence of the relaxed equilibrium configurations of the two NTCDA layers and the corresponding IS energies on two different approaches to treat dispersive van der Waals interactions and two DFT implementations has been analyzed in detail. From a methodological point of view it is found that the energies of the surface state and IS are very dependent on the specifics of the calculations (e.g., through the different equilibrium lattice parameter with different computational approaches) and are very hard to converge (e.g., with respect to the number of layers in the slab). However, the errors in the energies of the surface state $E_{S S}$ and interface state $E_{I S}$ tend to cancel, and the relative position of the IS with respect to the SS, $\Delta E=E_{I S}-E_{S S}$, is a more robust outcome of the calculation and provides the best way to make a comparison with experimental information, especially since $E_{S S}$ is known very accurately from the experiments.
Two-photon-photoemission results provide accurate measurements of the energies of the IS, giving values of $\Delta E$ of $\sim 490$ and $\sim 580 \mathrm{meV}$, respectively, for the compressed and relaxed phases. These results are in reasonable agreement with the computed values. However, DFT calculations using the optimized geometries are unable to reproduce the counterintuitive result that $\Delta E$ is higher for $\mathrm{r}-\mathrm{ML}$, in spite of its lower coverage $\Theta$. DFT calculations predict that $\Delta E$ is roughly the same for both r-ML and c-ML, with $E_{I S}$ only $\sim 20 \mathrm{meV}$ higher for the compressed layer. This small effect of the coverage difference can be understood from the higher average position of the molecules over the $\operatorname{Ag}(111)$ surface in the c-ML phase than in the r-ML phase. This also points to this height difference being the explanation for the experimental observation of a higher $E_{I S}$ for r-ML.

In order to arrive at a fundamental and thorough understanding of this interrelationship and in an effort to bring together structural and spectroscopic information, the dependence of $E_{I S}$ on vertical bonding distances $d_{\perp}$ and the surface coverage $\Theta$ has been analyzed. While the coverage contributes substantially to determining the value of $\Delta E$ (especially for vertical bonding distances $d_{\perp} \leqslant 3.1 \AA$ ), the impact of the molecular height $d_{\perp}$ is even larger in the relevant range. Our calculations show that height differences on the order of $d_{\perp}=0.1 \AA$ A. i.e., consistent with the experimental information available for the geometries of the r-ML and c-ML phases, are sufficient to compensate for the effect of the coverage difference and explain an $E_{I S}$ that is higher for r-ML than for c-ML.

Given the fact that the energetic position of $E_{I S}$ for molecular layers on metal substrates depends crucially on various geometric as well as electronic properties (of both 
the molecule and the substrate surface layer), the good agreement found between the theoretically calculated interface energies and experimentally observed values is exceptional. This underlines the thorough understanding of molecule-metal interactions and the high accuracy that DFT methodologies are acquiring which, in particular, benefit from the inclusion of advanced van der Waals correction schemes.

\section{ACKNOWLEDGMENTS}

This work is a project of the SFB 1083 "Structure and Dynamics of Internal Interfaces" funded by the Deutsche Forschungsgemeinschaft DFG (Germany). D.S.-P. acknowledges support from the Spanish MINECO (Spain), MAT201346593-C6-2-P.
[1] R. Temirov, S. Soubatch, A. Luican, and F. S. Tautz, Nature (London) 444, 350 (2006).

[2] C. H. Schwalb, S. Sachs, M. Marks, A. Schöll, F. Reinert, E. Umbach, and U. Höfer, Phys. Rev. Lett. 101, 146801 (2008).

[3] M. S. Dyer and M. Persson, New J. Phys. 12, 063014 (2010).

[4] N. L. Zaitsev, I. A. Nechaev, and E. V. Chulkov, J. Exp. Theor. Phys. 110, 114 (2010).

[5] M. Marks, N. L. Zaitsev, B. Schmidt, C. H. Schwalb, A. Schöll, I. A. Nechaev, P. M. Echenique, E. V. Chulkov, and U. Höfer, Phys. Rev. B 84, 081301 (2011).

[6] N. L. Zaitsev, I. A. Nechaev, P. M. Echenique, and E. V. Chulkov, Phys. Rev. B 85, 115301 (2012).

[7] S. S. Tsirkin, N. L. Zaitsev, I. A. Nechaev, R. Tonner, U. Höfer, and E. V. Chulkov, Phys. Rev. B 92, 235434 (2015).

[8] S. K. M. Henze, O. Bauer, T.-L. Lee, M. Sokolowski, and F. S. Tautz, Surf. Sci. 601, 1566 (2007).

[9] O. Bauer, G. Mercurio, M. Willenbockel, W. Reckien, C. Heinrich Schmitz, B. Fiedler, S. Soubatch, T. Bredow, F. S. Tautz, and M. Sokolowski, Phys. Rev. B 86, 235431 (2012).

[10] A. Hauschild, K. Karki, B. C. C. Cowie, M. Rohlfing, F. S. Tautz, and M. Sokolowski, Phys. Rev. Lett. 94, 036106 (2005).

[11] J. Ziroff, P. Gold, A. Bendounan, F. Forster, and F. Reinert, Surf. Sci. 603, 354 (2009).

[12] M. C. E. Galbraith, M. Marks, R. Tonner, and U. Höfer, J. Phys. Chem. Lett. 5, 50 (2014).

[13] M. Marks, A. Schöll, and U. Höfer, J. Electron Spectrosc. Relat. Phenom. 195, 263 (2014).

[14] U. Stahl, D. Gador, A. Soukopp, R. Fink, and E. Umbach, Surf. Sci. 414, 423 (1998).

[15] L. Kilian, U. Stahl, I. Kossev, M. Sokolowski, R. Fink, and E. Umbach, Surf. Sci. 602, 2427 (2008).

[16] J. Stanzel, W. Weigand, L. Kilian, H. L. Meyerheim, C. Kumpf, and E. Umbach, Surf. Sci. 571, L311 (2004).

[17] C. Stadler, S. Hansen, A. Schöll, T.-L. Lee, J. Zegenhagen, C. Kumpf, and E. Umbach, New J. Phys. 9, 50 (2007).

[18] A. Bendounan, F. Forster, A. Schöll, D. Batchelor, J. Ziroff, E. Umbach, and F. Reinert, Surf. Sci. 601, 4013 (2007).

[19] C. R. Braatz, G. Öhl, and P. Jakob, J. Chem. Phys. 136, 134706 (2012).
[20] R. Tonner, P. Rosenow, and P. Jakob, Phys. Chem. Chem. Phys. 18, 6316 (2016).

[21] C. R. Braatz, T. Esat, C. Wagner, R. Temirov, F. S. Tautz, and P. Jakob, Surf. Sci. 643, 98 (2016).

[22] S. Sachs, C. H. Schwalb, M. Marks, A. Schöll, F. Reinert, E. Umbach, and U. Höfer, J. Chem. Phys. 131, 144701 (2009).

[23] F. Reinert, G. Nicolay, S. Schmidt, D. Ehm, and S. Hüfner, Phys. Rev. B 63, 115415 (2001).

[24] R. Paniago, R. Matzdorf, G. Meister, and A. Goldmann, Surf. Sci. 336, 113 (1995).

[25] D. Sánchez-Portal, P. Ordejón, E. Artacho, and J. M. Soler, Int. J. Quantum Chem. 65, 453 (1997).

[26] J. M. Soler, E. Artacho, J. D. Gale, A. García, J. Junquera, P. Ordejón, and D. Sánchez-Portal, J. Phys. Condens. Matter 14, 2745 (2002).

[27] G. Kresse and J. Furthmüller, Comput. Mater. Sci. 6, 15 (1996).

[28] N. Troullier and J. L. Martins, Phys. Rev. B 43, 1993 (1991).

[29] P. E. Blöchl, Phys. Rev. B 50, 17953 (1994).

[30] G. Kresse and D. Joubert, Phys. Rev. B 59, 1758 (1999).

[31] J. Klimes, D. R. Bowler, and A. Michaelides, J. Phys. Condens. Matter 22, 022201 (2010).

[32] J. Klimes, D. R. Bowler, and A. Michaelides, Phys. Rev. B 83, 195131 (2011).

[33] S. Grimme, J. Antony, S. Ehrlich, and H. Krieg, J. Chem. Phys. 132, 154104 (2010).

[34] S. Grimme, S. Ehrlich, and L. Goerigk, J. Comp. Chem. 32, 1456 (2011).

[35] J. P. Perdew, K. Burke, and M. Ernzerhof, Phys. Rev. Lett. 77, 3865 (1996).

[36] S. Garcia-Gil, A. Garcia, N. Lorente, and P. Ordejon, Phys. Rev. B 79, 075441 (2009).

[37] A. Schöll, Y. Zou, T. Schmidt, R. Fink, and E. Umbach, J. Phys. Chem. B 108, 14741 (2004).

[38] G. I. Csonka, J. P. Perdew, A. Ruzsinszky, P. H. T. Philipsen, S. Lebegue, J. Paier, O. A. Vydrov, and J. G. Angyan, Phys. Rev. B 79, 155107 (2009). 